\title{
Hidatidosis en la infancia: Albendazol en su tratamiento médico y quirúrgico
}

\author{
ISABEL NOEMÍ H. ${ }^{1}$, ALEJANDRO VIOVY A. ${ }^{1}$, ROCÍO ZAMORANO P. ${ }^{2}$, ÁNGEL BLANCO M. ${ }^{3}$, \\ DANIEL REVELLO P. ${ }^{2}$, MLADEN VOJKOVIC L. ${ }^{2}$ y JOSÉ LUIS CERVA C. ${ }^{4}$.
}

\section{Hydatid disease in childhood: Albendazole in its medical and surgical treatment}

The aim of this study was to observe the response of hydatid disease in children to albendazole, either combined with or without surgery. The study was performed at Dr. L. Calvo Mackenna Hospital, between 1997 and 2000, in 15 patients. Thirteen patients received post surgery treatment because of daughter cyst finding and 2 received chemotherapy only. Albendazole was given orally (dose: $5 \mathrm{mg} / \mathrm{kg}$ bid for a mean time of 25 days) in 1 to 5 courses. Treatment evaluation was based on changes in cyst morphology, sonography and computed tomography, hematological and serological analysis. Seven patients had full response; the other had clinical and serological improvement. According with previously published experience, albendazole was efficient as a paliative pharmacological treatment instead of surgical procedure, in case of non-practicable hydatid cyst surgery. Moreover, albendazole is useful in case of multiple daughter cysts, well tolerated and reduced side effects in the follow-up.

Key words: Hydatid disease, Echinococcus granulosus, albendazole.

\section{Introducción}

La hidatidosis humana es la infección parasitaria zoonótica producida por el estado larval de céstodos del género Echinococcus. La especie que existe en Chile es E. granulosus cuyo hospedero definitivo es el perro y otros cánidos, mientras que los ganados ovino, bovino, caprino, porcino, etc corresponden a los huéspedes intermediarios habituales; el hombre constituye un hospedero intermediario accidental. El mecanismo de infección de la hidatidosis es la ingestión de huevos embrionados de E. granulosus, con el consecuente desarrollo de la hidátide en cualquier órgano de la economía, predominando las locali- zaciones hepática y pulmonar. El metacéstodo más la reacción fibrosa en su periferia (adventicia), generada por el propio organismo afectado, se denomina quiste hidatídico $(\mathrm{QH})$.

En el hombre el QH es generalmente único, a diferencia de lo que ocurre en el ganado; en las personas adultas su crecimiento es lento, estimándose su velocidad en aproximadamente $1 \mathrm{~cm}$ por año, lo que aparentemente es algo mayor en niños ${ }^{1}$.

En su evolución, el QH puede sufrir procesos degenerativos o involutivos como la calcificación; además puede romperse originando serias complicaciones: inmediatas, a corto y mediano plazo.

\footnotetext{
Hospital Luis Calvo Mackenna y Facultad de Medicina, Universidad de Chile:

Departamento de Pediatría, Campus Oriente.

Estudiante de Medicina.

Servicio de Cirugía Infantil.

Laboratorio de Parasitología.
}

Trabajo realizado en la Unidad de Parasitología del Departamento de Pediatría. Campus Oriente. Universidad de Chile. Hospital Luis Calvo Mackenna. 
La mayoría de las personas que padecen de hidatidosis son asintomáticas en los primeros años. La enfermedad clínica aparece cuando el $\mathrm{QH}$ adquiere un tamaño significativo y suele ser el resultado de la compresión de distintos órganos del hospedero. Generalmente, esta infección se adquiere en la infancia, pero las manifestaciones clínicas aparecen varios años después ${ }^{2}$.

Aunque el tratamiento de elección de la hidatidosis es quirúrgico, existen situaciones en que los derivados benzomidazólicos han mostrado utilidad como terapia alternativa: en caso de quistes inextirpables o terapia complementaria a la cirugía, especialmente ante quistes fértiles fisurados o rotos, antes o durante la operación. De estos antihelmínticos, el más usado es albendazol por su mejor biodisponibilidad en los tejidos, menores dosis a administrar, buena tolerancia y periodos de administración breves, en comparación con otros, como mebendazol ${ }^{3}$. Este tratamiento médico de la hidatidosis ha sido evaluado favorablemente en pacientes adultos y, según la literatura, ha demostrado ser especialmente efectivo en $\mathrm{QH}$ con un diámetro menor o igual a $5 \mathrm{~cm}^{4}$.

Con el objetivo de observar el efecto del albendazol en pacientes pediátricos con hidatidosis, se consideró relevante analizar en nuestro medio y en forma prospectiva, la respuesta a albendazol en niños con hidatidosis, independiente de su localización, ya sea como complemento del tratamiento quirúrgico, o bien como terapia exclusiva en caso de $\mathrm{QH}$ inextirpables o ante el hallazgo de quistes con un diámetro menor o igual a $3 \mathrm{~cm}$ que no provocan sintomatología.

\section{Pacientes y Métodos}

Desde abril de 1997 hasta diciembre del 2000, se estudiaron en forma prospectiva 15 pacientes, en quienes que se pesquisó al menos un $\mathrm{QH}$, ya sea en forma ambulatoria o durante una hospitalización, y que consultaron en forma consecutiva al Hospital Luis Calvo Mackenna. Por tratarse de un hospital pediátrico público todos los pacientes eran menores de 15 años. Una vez hecho el diagnóstico de hidatidosis a través de criterios clínicos, serológicos e imagenológicos, el caso se ingresaba a un protocolo de seguimiento, por un período que en promedio fue de 3 años 3 meses. Los niños fueron tratados con albendazol en dosis de $10 \mathrm{mg} / \mathrm{kg} /$ día fraccionado cada 12 horas, indicado por ciclos de 21 a 28 días, según lo recomendado por diversos autores ${ }^{5,6}$, administrado por vía oral, a partir de las 48 horas poste- riores a la cirugía. El tratamiento médico exclusivo se aplicó cuando la extirpación involucraba inminente riesgo vital para el paciente o, puntualmente, en $\mathrm{QH}$ pequeños únicos $(<3 \mathrm{~cm}$ en su diámetro mayor) que constituyeron hallazgos imagenológicos. Previo al inicio de albendazol y en el curso de su administración, los niños eran controlados clínicamente y con exámenes de laboratorio (hemograma con recuento de reticulocitos, pruebas de función hepática y renal) cada 7 a 10 días durante los ciclos, y cada 30 días en los periodos de "descanso". Se efectuó además, estudios por imágenes y serológicos cada 3 meses, hasta la resolución del caso. La monitorización de este tratamiento se realizó en la Policlínica de Parasitología Infantil del mencionado hospital.

Los criterios diagnósticos utilizados fueron los siguientes:

- Clínicos: Síntomas generales como compromiso del estado general, síndrome febril (por rotura e infección secundaria), anorexia y urticaria fugaz repetitiva. Síntomas específicos según localización ${ }^{7}$.

- Serológicos: Técnica de enzimo inmuno ensayo (ELISA IgG) positiva para hidatidosis humana $^{8,9}$.

- Imagenológicos: A todos se les realizó radiografía de tórax, ecotomografía abdominal y ecocardiografía. En caso de compromiso del SNC se practicó además, TAC encefálica.

En los pacientes sometidos a cirugía se confirmó el diagnóstico por biopsia de la pieza operatoria y se estudió la fertilidad de los quistes.

La respuesta a tratamiento se evaluó según la evolución de los mismos criterios. Se consideró que hubo mejoría del paciente al darse alguna (s) de las siguientes condiciones: remisión de las manifestaciones clínicas, negativización de los títulos serológicos y signos imagenológicos de compromiso vital de los quistes: involución total o disminución significativa de su tamaño, desarrollo de focos hiperecogénicos en su interior, aumento en la densidad del líquido hidatídico, engrosamiento o depósitos de calcio en la pared y/o desprendimiento de las membranas ${ }^{10}$. En caso de cumplir estos tres criterios simultáneamente se asumió que el niño estaba curado, por el contrario, al no cumplir ninguno de ellos, el tratamiento habría fracasado.

\section{Resultados}

De los 15 pacientes estudiados 8 fueron de sexo masculino y 7 femenino, las edades en el 
momento del diagnóstico fluctuaron entre 4 meses y 14 años 4 meses con un promedio de 8 años 1 mes. Nueve vivían en un ambiente rural y 6 procedían de zonas urbanas (Tabla 1). En 3 se obtuvo el antecedente de haber tenido contacto con perros que fueron alimentados con "bolsas de agua" (QH presentes en las vísceras del ganado). El ELISA (IgG) para hidatidosis resultó positivo en todos los casos. Del total de pacientes, en 13 el QH fue único y dos resultaron portadores de 2 quistes cada uno. La localización de los QH fue: 9 hepáticos, 5 pulmonares, 2 cerebrales y 1 inguinal. El volumen osciló entre 3 y $300 \mathrm{cc}$ con un promedio de 49 cc, observándose en la localización pulmonar los quistes de mayor tamaño $(\overline{\mathbf{x}}$ $=74,2 \mathrm{cc}$ ) (Tabla 2). El estudio de fertilidad fue positivo para 10 , negativo para 4 y no se precisó en 3 , ya que al no ser extirpados, obviamente, no se enviaron a estudio anátomo patológico. No existió correlación entre el tamaño de los quistes y la fertilidad de éstos $(r=0,137)$ (Tabla $3)$.

Los dos pacientes que no fueron intervenidos quirúrgicamente, recibieron tratamiento farmacológico exclusivo: un caso correspondió a una niña de 5 años con un QH en cada lóbulo hepático -considerados fuera de solución quirúrgica- y el otro se trató de un niño de 6 años portador de un pequeño quiste $(3 \times 2 \mathrm{~cm})$ en el lóbulo hepático derecho, escasamente sintomático. En el resto se procedió a la extirpación quirúrgica evidenciándose rotura en la mitad de los $\mathrm{QH}$ intervenidos.

El número de curas con albendazol para cada niño osciló entre 1 y 5 (2,3 en promedio) con un promedio de duración de 25 días (rango 17-30 días). Sólo en un paciente este benzimidazólico se asoció a la aparición de fiebre, artralgias, vómitos y eritema nódulo-papular, lo que remitió rápidamente una vez suspendido el fármaco.

De acuerdo a los criterios establecidos, todos los pacientes respondieron satisfactoriamente a la terapia instaurada: 7 curaron (Tabla 4) y los 8 restantes (Tabla 5) presentaron una notable mejoría clínica, además de negativización serológica en 3, disminución significativa de los títulos del ELISA (IgG) para hidatidosis en 5 y signos imagenológicos categóricos de compromiso vital del QH en 2.

Todos los niños tuvieron una ecocardiografía informada como normal y durante el periodo de seguimiento ningún caso presentó hidatidosis secundaria.
Tabla 1. Características de los pacientes

\begin{tabular}{cccc}
\hline Pacientes & Edad de diagnóstico & Sexo & Procedencia \\
\hline 1 & 2 años 9 meses & Masculino & Rural \\
2 & 14 años 4 meses & Femenino & Rural \\
3 & 6 años & Masculino & Urbana \\
4 & 4 meses & Femenino & Urbana \\
5 & 7 años 6 meses & Masculino & Urbana \\
6 & 13 años 4 meses & Masculino & Rural \\
7 & 10 años 4 meses & Femenino & Rural \\
8 & 5 años & Femenino & Rural \\
9 & 11 años 9 meses & Masculino & Rural \\
10 & 5 años 10 meses & Femenino & Rural \\
11 & 11 años 10 meses & Masculino & Urbana \\
12 & 10 años & Masculino & Rural \\
13 & 9 años 6 meses & Masculino & Rural \\
14 & 4 años 4 meses & Femenino & Urbana \\
15 & 8 años 9 meses & Femenino & Urbana \\
& Promedio: & Femenino: 7 & Rural: 9 \\
& 8 años 1 mes & Masculino: 8 & Urbana: 6 \\
\hline
\end{tabular}

Tabla 2. Volumen de los quistes hidatídicos según ubicación

\begin{tabular}{lcc}
\hline Localización & $\begin{array}{c}\text { Número } \\
(\mathbf{n}=\mathbf{1 7})\end{array}$ & $\begin{array}{c}\text { Volumen } \\
(\overline{\mathbf{x}} \text { en } \mathbf{c c} .)\end{array}$ \\
\hline Pulmón & 5 & 74,2 \\
Hígado & 9 & 45 \\
Cerebro & 2 & 23 \\
Inguinal & 1 & 6 \\
\hline
\end{tabular}

$\chi^{2} \quad \mathrm{p}<0,01$

\section{Discusión}

La alta morbilidad en ciertas regiones, la potencial letalidad en caso de no ser tratada oportunamente, la mayor frecuencia del diagnóstico en adultos jóvenes -edad de máxima productividad laboral- y los costos por concepto de diagnóstico y tratamiento (hospitalizaciones y cirugías) incluyendo las recidivas, destacan la importancia epidemiológica de la hidatidosis ${ }^{11}$. Además, esta zoonosis, por ser prevalente en zonas agrícolas y ganaderas, representa un importante problema en medicina veterinaria por las consecuencias económicas que tiene en la productividad pecuaria ${ }^{12}$.

En América Latina, los países con los índices más elevados de infección humana son Argentina, Chile, Uruguay, Brasil, Colombia, Bolivia y Paraguay. Oficialmente, según el registro de Enfermedades de Notificación Obligatoria del Ministerio de Salud, la tasa global de incidencia en 
Tabla 3. Características de los quistes hidatídicos

\begin{tabular}{|c|c|c|c|c|}
\hline Pacientes & Tamaño (cm) & \multicolumn{2}{|c|}{ Localización (*) } & Fertilidad \\
\hline 1 & $2 \times 3$ & \multicolumn{2}{|c|}{ Inguinal izquierdo } & Sí \\
\hline 2 & $12 \times 10 / 3 \times 4$ & \multicolumn{2}{|c|}{ LPSD / LHD } & Sí / Sí \\
\hline 3 & $3 \times 2$ & \multicolumn{2}{|c|}{ LHD } & -- \\
\hline 4 & $3 \times 4$ & \multicolumn{2}{|l|}{ LHD } & Sí \\
\hline 5 & $10 \times 12$ & \multicolumn{2}{|l|}{ LHI } & No \\
\hline 6 & $5 \times 3$ & \multicolumn{2}{|l|}{ LHI } & Sí \\
\hline 7 & $12 \times 10$ & \multicolumn{2}{|c|}{ LHD } & Sí \\
\hline 8 & $6 \times 4 / 3 \times 2$ & \multicolumn{2}{|c|}{ LHD / LHI } & -- \\
\hline 9 & $7 \times 6$ & \multicolumn{2}{|c|}{ LCFD } & No \\
\hline 10 & $15 \times 20$ & \multicolumn{2}{|l|}{ LPID } & Sí \\
\hline 11 & $2 \times 2$ & \multicolumn{2}{|l|}{ LCFD } & Sí \\
\hline 12 & $4 \times 4$ & \multicolumn{2}{|l|}{ LPSI } & No \\
\hline 13 & $6 \times 10$ & \multicolumn{2}{|l|}{ LHD } & Sí \\
\hline 14 & $3 \times 1$ & \multicolumn{2}{|l|}{ LPID } & No \\
\hline \multirow[t]{8}{*}{15} & $6 \times 3$ & \multicolumn{2}{|l|}{ LPID } & Sí \\
\hline & & LPSI & $=1$ & Fértiles $\quad=10$ \\
\hline & & LPSD & $=1$ & No fértiles $=4$ \\
\hline & & LPID & $=3$ & No extirpado $=3$ \\
\hline & & LHD & $=6$ & \\
\hline & & LHI & $=3$ & \\
\hline & & LCFD & $=2$ & \\
\hline & & Inguina & $=1$ & \\
\hline
\end{tabular}

Correlación tamaño $v / s$ fertilidad de QH r $=0,137$ ( NS). (*) LPSI: lóbulo pulmonar superior izquierdo; LPSD: lóbulo pulmonar superior derecho; LPID: lóbulo pulmonar inferior derecho; LHD: lóbulo hepático derecho; LHI: lóbulo hepático izquierdo; LCFD: lóbulo cerebral frontal derecho.

Tabla 4. Características de los pacientes que curaron

\begin{tabular}{llccc}
\hline Edad al diagnóstico & Localización del quiste $\left(^{*}\right)$ & Cirugía & Rotura quiste & $\mathbf{N}^{\circ}$ de curas \\
\hline 2 años 9 meses & Inguinal izquierdo & Sí & Sí & 4 \\
9 años 6 meses & LHD & Sí & Sí & 3 \\
5 años & LHD / LHI & No & No & 2 \\
11 años 9 meses & LCFD & Sí & Sí & 2 \\
11 años 10 meses & LCFD & Sí & No & 1 \\
8 años 9 meses & LPID & Sí & Sí & 3 \\
10 años 4 meses & LHD & & \\
\hline
\end{tabular}

(*): LPID: lóbulo pulmonar inferior derecho; LHD: lóbulo hepático derecho; LHI: lóbulo hepático izquierdo; LCFD: lóbulo cerebral frontal derecho.

Tabla 5. Características de los pacientes que mejoraron

\begin{tabular}{lcccc}
\hline Edad al diagnóstico & Localización de quiste & Cirugía & Rotura quiste & $\mathbf{N}^{\circ}$ de curas \\
\hline 5 años 10 meses & LPID & Sí & No & 1 \\
14 años 4 meses & LHD/ LPSD & Sí & No & 3 \\
4 meses & LHD & Sí & Sí & 2 \\
13 años 4 meses & LHI & Sí & Sí & 5 \\
4 años 4 meses & LPID & Sí & Sí & 1 \\
10 años & LPSI & Sí & No & 1 \\
6 años & LHD & No & -- & 5 \\
7 años 6 meses & LHI & Sí & No & 1 \\
\hline
\end{tabular}

(*): LPSI: lóbulo pulmonar superior izquierdo; LPSD: lóbulo pulmonar superior derecho; LPID: lóbulo pulmonar inferior derecho; LHD: lóbulo hepático derecho; LHI: lóbulo hepático izquierdo. 
Chile se ha mantenido relativamente estable durante los últimos 12 años; en 2002 fue de 2,16 enfermos por cada 100.000 habitantes, observándose la mayor notificación de casos en las regiones VIII, X y XI, con tasas aproximadas de 13,11 y 40 por 100.000 habitantes, respectivamente $^{13}$. Sin embargo, diversos estudios demuestran que, al comparar los egresos hospitalarios con el diagnóstico de hidatidosis de distintos servicios de cirugía y los casos efectivamente notificados, existe una evidente subnotificación ${ }^{14}$. Es más, al determinar la tasa de infectados mediante estudios de seroprevalencia, ésta aumenta notablemente a 136/100.000 habitantes, a nivel nacional ${ }^{15}$, lo que es concordante con estudios de autopsias efectuadas en individuos no seleccionados del Servicio Médico-Legal de Santia$\mathrm{go}^{16}$.

Se estima que aproximadamente $20 \%$ de los enfermos con hidatidosis corresponden a niños y que la mayoría de ellos se encuentra en fase asintomática. En el Hospital Luis Calvo Mackenna de Santiago, la hidatidosis es una causa de derivación desde áreas periurbanas y rurales, representando 3\% del total de consultas anuales.

El tratamiento de elección de la hidatidosis es la cirugía, con el costo familiar, social y económico que involucra, además del escolar por tratarse de niños. El disponer de un tratamiento médico efectivo, ambulatorio, de fácil administración, bien tolerado y económicamente accesible, representa un importante avance en el manejo racional de esta grave patología. En este sentido, albendazol, que actúa sobre la capa germinativa de la hidátide, inhibiendo la síntesis de la tubulina citoplasmática, bloqueando la absorción de glucosa por parte del parásito y produciendo una alteración degenerativa en el sistema retículo endoplásmico y de las mitocondrias ${ }^{17}$, constituiría un excelente quimioterápico para ser utilizado como tratamiento complementario a la cirugía con el fin de evitar la diseminación secundaria a partir de QH fértiles o como terapia alternativa en caso de $\mathrm{QH}$ fuera del alcance quirúrgico o en etapa incipiente ${ }^{18}$.

Con la dosis utilizada se apreció una buena tolerancia y escasos efectos secundarios en esta serie clínica prospectiva.

Los pacientes curados recibieron un número variable de ciclos con albendazol, entre 1 y 4 . No se logró definir un número óptimo uniforme de curas que fuera comprobadamente eficaz, concluyéndose que en el complejo proceso de curación, se deben considerar diversos otros factores dependientes de la interacción hospedero-parásitoambiente, aparte del número de ciclos de terapia; esto último dependerá de la cuidadosa evaluación clínica integral y de los exámenes complementarios por parte del médico tratante.

A todos los pacientes que ingresaron al protocolo se les practicó una ecocardiografía la que resultó normal en $100 \%$ de los casos; estimamos que no es necesario realizar este examen en forma rutinaria para descartar compromiso cardíaco en individuos con hidatidosis de otra localización, salvo que la anamnesis y/o el examen físico lo sugieran ${ }^{19}$.

Ulteriores seguimientos y una mayor casuística son necesarios para evaluar la acción benéfica de los derivados benzoimidazólicos absorbibles, como el albendazol, en el tratamiento de la hidatidosis en la edad pediátrica. Se estima que el diagnóstico de hidatidosis en niños probablemente irá en aumento en la medida que mejore la accesibilidad a exámenes radiográficos, ecográficos y eventualmente serológicos que permitan hacer el diagnóstico precoz, en etapa de infección asintomática.

Además del interés en los aspectos curativos de la hidatidosis que motivaron este trabajo, es fundamental enfatizar la prevención primaria. Entre los factores que predisponen a la adquisición de esta enfermedad, se encuentran el desconocimiento del problema por parte de la población y la presencia de hábitos y costumbres que facilitan la transmisión de este parásito: alimentar a los perros con vísceras crudas y las condiciones sociales y ambientales que favorecen el fecalismo canino ${ }^{20}$. Al existir un ambiente propicio y conductas de riesgo comunes que contribuyen a la mantención del ciclo del parásito en la naturaleza y la infección humana, es importante, una vez hecho el diagnóstico de un caso índice, llevar a cabo un estudio familiar que, idealmente, debería incluir: evaluación clínica, serología específica (ELISA $\operatorname{IgG}$ ), radiografía de tórax y ecotomografía abdominal.

Se insiste en la necesidad de un programa activo de vigilancia epidemiológica, de educación comunitaria y de prevención sistemática nacional de la hidatidosis humana, enfocado especialmente aunque no exclusivamente, a los grupos de alto riesgo ${ }^{21}$.

\section{Resumen}

El objetivo de este trabajo fue observar la respuesta a albendazol en niños con hidatidosis como terapia exclusiva o complementaria al tratamiento quirúrgico. Entre 1997 y 2000 se enrolaron 15 casos que consultaron consecutivamente al Hospital Luis Calvo Mackenna con diagnóstico de quiste hida- 
tídico. En 13 se realizó cirugía y tratamiento médico asociado. En 2 el tratamiento fue sólo farmacológico. El albendazol se administró en dosis de $10 \mathrm{mg} / \mathrm{kg} / \mathrm{día}$ por un promedio de 25 días (entre 1 y 5 curas). Los niños fueron controlados cada 3 meses con evaluación clínica, determinación de parámetros bioquímicos y hematológicos, además de los estudios imagenológicos procedentes (radiografía, ecotomografía, tomografía axial computarizada) y serológicos (ELISA IgG específica). Siete pacientes curaron y el resto mostró notable mejoría (clínica, serológica e imagenológica). Debe destacarse el albendazol como terapia paliativa, curativa y como complemento a la cirugía. Con las dosis y períodos utilizados hubo buena tolerancia, escasos efectos adversos y adecuada adhesión al tratamiento.

\section{Bibliografía}

1.- Larrieu E, Frider B. Human cystic echinococcosis: contributions to the natural hystory of the disease. Ann Trop Med Parasitol 2001: 95: 679-87.

2.- Sapunar J. En: Parasitología Médica, A Atías, ed. Capítulo 39; Hidatidosis. Primera edición, Mediterráneo Ltda. Santiago, Chile 1999; 253-338.

3.- Atias A. El tratamiento médico de la hidatidosis. Parasitol al Día 1993; 17: 153-7.

4.- Apt W, Pérez C, De Rycke P. Tratamiento no convencional de la hidatidosis humana. Rev Méd Chile 1996; 124: 1385-9.

5.- Ayles H, Corbett E, Taylor I et al. A combined medical and surgical approach to hydatid disease: 12 years experience at the Hospital for Tropical Diseases, London. Ann R Coll Surg Engl 2002; 84: 100-5.

6.- Todorov T, Vutova K, Mechcov G et al. Experience in the chemotherapy of severe, inoperable echinococcosis in man. Infection.1992; 20: 19-24.

7.- Noemí I, Benedetto S, Ahumada O et al. Hidatidosis en pediatría. Parasitol al Día 1991; 15: 108-13.

8.- Contreras M, Gallo S, Salinas P, Sapunar J, Sandoval L, Solis F. Evaluación de la ELISA IgG, usando antígeno purificado en el diagnóstico de la hidatidosis humana. Bol Chil Parasitol 1994; 49: 24-30.

9.- Jercic M I, Astorga B. Estandarización de la técnica de enzimo inmuno ensayo para el diagnóstico de la hidatidosis humana. Rev Chil Tec Médica 1998; 18: 31-2.

10.- Todorov T, Vutova K, Mechcov G et al. Evaluation of response to chemotherapy of human cystic echinococcosis. Br J Radiol 1990; 523-31.

11.- Aliaga F, Oberg C. Epidemiología de la hidatidosis humana en la IX Región de la Araucanía, Chile. 19911998. Bol Chil Parasitol 2000; 55: 54-8.

12.- Serra I, Araneda J, Araya C, Serra V. Análisis regional de la hidatidosis humana y animal en Chile. 19891993. Bol Chil Parasitol 1994; 51: 3-12.

13.- Sotomayor V. Enfermedades de Notificación Obligatoria: Zoonosis y Chagas. El Vigía 2002; 6: 28.

14.- Serra I, Araneda J, Araya C, Serra V. Situación de la hidatidosis humana en Chile. Dos propuestas de corrección de subnotificación. Rev Méd Chile 1995; 123: 659-9.

15.- Schenone H, Contreras M, Salinas P. Hidatidosis humana en Chile. Seroprevalencia y estimación del número de personas infectadas. Bol Chil Parasitol 1999; 54: 70-3.

16.- Guerra F, Cortes M, Araneda T et al. Algunas características epidemiológicas de la hidatidosis y cisticercosis en cadáveres de personas autopsiadas en la Región Metropolitana, Chile. Bol Chile Parasitol 1985; 40: 38-41.

17.- Stieger V, Cotting J, Reichen J. Albendazole treatment of echinococcosis in humans: effects on microsomal metabolism and drug tolerance. Clin Pharmacol Ther 1990; 47: 347-53.

18.- Poyanli A, Akan K, Poyalinli O, Sencer S, Sayarak H. Primary hydatid disease of the femur: unsuspected and incidental MRI findings with long term curative results on medical treatment alone. Skeletal Radiol 2001; 30: 656-8.

19.- Viovy A, Noemí I, Soler P, Gómez O, Haecker S, Zilleruelo R. Hidatidosis cardíaca: Caso clínico pediátrico complicado de embolia cerebral tratado exitosamente con cirugía cardíaca. Rev Chil Cardiol 1994; 13: 127-30.

20.- Larrieu E L, Costa M T, Del Carpio M, Moquillans K Y, Bianchi G, Yadon Z E. Case control study of risk factors for cystic echinococcosis among the children of Rio Negro province Argentina, Ann. Trop Med Parasitol 2002; 96: 43-52.

21.- Busel D. Epidemiología de la hidatidosis: determinación de grupos de alto riesgo y educación como una alternativa racional. Rev Méd Sur 1991; 16: 10-1.

Correspondencia a:

Isabel Noemí Hauck

E-mail: ihauck@med.uchile.cl 